\title{
Mathematics Education and the Objectivist Programme in HPS
}

\author{
Eduard Glas
}

Published online: 1 January 2012

(C) The Author(s) 2011. This article is published with open access at Springerlink.com

\begin{abstract}
Using history of mathematics for studying concepts, methods, problems and other internal features of the discipline may give rise to a certain tension between descriptive adequacy and educational demands. Other than historians, educators are concerned with mathematics as a normatively defined discipline. Teaching cannot but be based on a pre-understanding of what mathematics 'is' or, in other words, on a normative (methodological, philosophical) view of the identity or nature of the discipline. Educators are primarily concerned with developments at the level of objective mathematical knowledge, that is: with the relations between successive theories, problems and proposed solutions-relations which are independent of whatever has been the role of personal or collective beliefs, convictions, traditions and other historical circumstances. Though not exactly 'historical' in the usual sense, I contend that this 'objectivist' approach does represent one among other entirely legitimate and valuable approaches to the historical development of mathematics. Its retrospective importance to current practitioners and students is illustrated by a reconstruction of the development of Eudoxus's theory of proportionality in response to the problem of irrationality, and the way in which Dedekind some two millennia later almost literally used this ancient theory for the rigorous introduction of irrational numbers and hence of the real number continuum.
\end{abstract}

\section{Introduction}

Mathematics belongs to the very heart of modern science and technology, and hence of our science and technology-based culture. It goes without saying, therefore, that learning about the history of this characteristic aspect of our culture is of great educational value. There are a great many reasons indeed for incorporating history of mathematics in education, either as part of general cultural studies or more specifically in the teaching of mathematics proper (overview in Fauvel and Van Maanen 2000; cf. also: Jankvist 2009, 2010;

\footnotetext{
E. Glas $(\bowtie)$

Department of Mathematics, Delft University of Technology, Mekelweg 4,

2628 CD Delft, The Netherlands

e-mail: e.glas@tudelft.nl
} 
Charalambous et al. 2009; Gulikers and Blom 2001). As to the first aspect, learning about the history of mathematics may be a way of humanizing the discipline, showing it to be a human enterprise, prone to anything human, and fulfilling various social and cultural functions in the course of its development. As to the second aspect, history of mathematics may be invoked as a powerful means for introducing and studying concepts, methods, problems and other internal features of the discipline. In the latter case we are concerned primarily with learning from the history of mathematics, and it is this concern that the present paper is about.

It is not my intention to implement any educational programme for use in the classroom. My modest aim is to argue that there is room for a problem-driven, objectivist approach that focuses expressly on the situational logic of the objective problem situation as the rational ground for conceptual development in mathematics. Though mathematics surely is a social and cultural product, it is not merely a social and cultural product: it has an objective content from which arise problems that are independent from the practice that produced it. It is worthwhile to reconstruct the objective aspects of the contemporary problem situations as one among other equally legitimate and valuable approaches to the history of mathematics-depending, of course, on one's educational goals.

\section{2 'Real' Versus 'Rationally Reconstructed' History}

The very first thing to be learned from the history of mathematics is the inadequacy of the cumulative model of mathematical development. Although expanding the body of proven propositions is certainly important, mathematicians are not interested just in truths (much less in truisms), but in truths that provide answers to problems that are challenging, interesting and fertile for further research. In other words: the development of mathematics turns on the heuristic potential rather than the mere truth of propositions. It is only in relation to a certain problem situation - a problem together with a background of available theorems, arguments, criticisms, etc.-that the rationality and comprehensibility of developments can be judged. Therefore, in order to learn from the mathematics of bygone ages, we have to reconstruct the objective problem situations that gave rise to certain concepts, problems and problem-solving methods.

Such rational reconstructions are of limited use for understanding the so-called 'real' history of mathematics, yet are indispensable for learning from it. Indeed, history by itself just tells us of more or less distant and exotic practices to which we have no access other than through some form of understanding of their continuity with present mathematical concerns. Abandoning any rational standpoint would land us in a relativism that would prevent us from distinguishing between genuine mathematics and charlatanism, and deprive us of any sense of continuity and direction. This may be alright as far as history per se is concerned, but it makes the relevance of the history of mathematics for our understanding of present-day mathematics highly questionable, especially to students. There is no learning from the past without some form of rational reconstruction.

Clearly, using a historical approach in studying concepts, methods, problems and other internal features of the discipline, may easily give rise to a certain tension between standards of descriptive adequacy and educational demands. It has been said, for instance, that such an approach forces one 'either to trivialise history or to distort it' (Fried 2001). In studying the mathematics of the past with the said educational intentions, one may tend to broaden the interpretation of old ideas, impermissibly linking them with modern, much more general notions and conceptions. The approach therefore is deemed to be intrinsically 
a-historical. Mathematics educators must in this view choose either to remain true to their commitment to modern mathematics and risk being unhistorical in their approach, or take a genuinely historical approach and risk spending time on things irrelevant to the mathematics they have to teach.

When history of mathematics is conceived of as an attempt to detect and explain the historical ordering, development, dissemination, and effects of ideas and intellectual traditions, then the said objections are certainly to the point. Other than historians, however, educators are concerned with 'mathematics' as a normatively defined category-hence with a normatively reconstructed history as opposed to a history of ideas. Teaching cannot but be based on a pre-understanding on the part of the teacher of what mathematics 'is' or, in other words, on a normative view of the identity or nature of the discipline. He or she has to reconstruct historical episodes according to a normative, that is: a methodological or philosophical, view, producing one way or another a 'rational reconstruction' of history.

I contend that as far as our goal is to learn about mathematical in-issues, we are concerned primarily with the objective aspects of historical developments. In contradistinction to history of mathematics as a history of mathematicians and their notions and conceptions, such an 'objectivist' reconstruction is not concerned with what the historical figures 'really' had in mind. It is concerned instead only with the relations between theories, problems, proposed solutions, criticisms, and so on, relations which are independent of whatever has been the role of personal or collective beliefs, convictions and the like. We are concerned, then, with mathematical knowledge as part of what Popper has dubbed the third world (or world-3), the man-made though semi-autonomous world of theories, problems and arguments in the objective sense (Popper 1972). I will develop these ideas somewhat further in the next section.

Though perhaps not 'historical' in the usual sense, I contend that the objectivist approach can be a useful study of the past, representing one among other entirely legitimate and valuable approaches to the history of mathematics. For the purpose of learning from the history of mathematics, we must focus on the development of mathematical knowledge in itself, taken impersonally, and not on the activities of individual mathematicians or their institutions. In order to acquire this understanding of the historical development of mathematical knowledge, we have to focus in particular on the contemporary objective problem situation and the available resources for tackling these problems. I will illustrate this with an example in Sect. 4.

\section{Objective Problems}

With Popper, we will call knowledge objective when it is articulated impersonally and is laid down in non-mental information bearers - for instance on paper or electronically-so as to be publicly accessible and debatable. What we call 'the body of knowledge' consists of the totality of theories, conjectures, criticisms, etc., that in this sense is objectively available.

Knowledge cannot be articulated, argued, debated, criticised, etc., without using language, and language presupposes public, impersonal means of expression. Without language there cannot be objective knowledge. Mathematics in particular may be regarded as the product of the development of discursive language into a critical instrument for the study of relations too complex for ordinary language to deal with effectively. Criticism and the articulation of theories are only possible in language, which gives them their objective character. Acquiring a language essentially means being able to grasp objective thought 
contents. The development of mathematics shows that with new linguistic means new kinds of facts and in particular new kinds of problems can be described and argued. As we shall see, Eudoxus's solution to the problem of irrationality is a nice example of the creation of new linguistic means to 'express the (previously) inexpressible'.

So the elements of the body of mathematical knowledge are of a linguistic nature and the relations between these elements are of a logical nature. One may for instance ask if a piece of mathematics is internally consistent and consistent with other parts of the body of mathematics, if the reasoning is correct, if it solves some relevant problem, etc. These are all objective questions in that they are concerned with the objective merits of a theory and have nothing to do with the mental states and processes of the persons who have this knowledge 'in their heads'. Objective knowledge also develops partially autonomously: our objectively articulated mathematical creations often bring with them entirely unintended and unexpected problems. By trying to solve these problems-which thus are not just our own creations-we produce new mathematical objects, problems and critical arguments. Unsolved problems within theories and well-formed but undecidable questions (such as Goldbach's conjecture or the continuum hypothesis) also are indications of the mind-independence and semi-autonomous development of the third world.

Of course, to stress the objective and partially autonomous dimension of mathematical knowledge is not to lose sight of the fact that it is created, discussed, evaluated, tested, and modified by human beings in a social environment. Mathematics is the product of a communal practice, but at the same time this product-the content of mathematicsbecomes partially autonomous from the practice that produced it.

History in the third world is the history of objective growth, and so the difference between this 'objectivist' approach and a history of ideas-which is focussed more on the second world of mental states and processes-is understandable. In particular, the objective content of a theory may be significantly greater than its development in human minds in the second world. As will be illustrated in the next section, the surplus content of Eudoxus's theory of proportionality, for instance, was exploited successfully by Dedekind for the rigorous introduction of the real number continuum.

\section{The Example of Irrationality}

Mathematical objects, the system of natural numbers in particular, may be regarded as evolutionary products of human language and human thought. For instance, as soon as the natural numbers had been created or invented, the distinctions between odd and even, and between prime and composite numbers, and the ensuing problem of what is called 'the irrationality of the square root of 2', came to exist objectively. It in fact constituted the objective problem situation that faced the Pythagorean arithmetical cosmology, in which it was assumed that ultimately all things in the world could be characterised by numbers and ratios between numbers. They had discovered that the diagonal and the side of a square are not measurable against each other; this is the famous discovery that the square root of 2 cannot be expressed as the ratio of two integers. How was this discovery made? We have Aristotle's report, which says that 'they prove that the side and diagonal of a square are incommensurable by showing that, if they would be commensurable, odd numbers would have to be equal to even numbers'.

This statement is the basis of a reconstitution of the Pythagorean proof that is highly relevant educationally. Let us assume that the diagonal and the side have a common measure, and that they stand in the ratio p:q (with $\mathrm{p}$ and $\mathrm{q}$ integers). Then, according to 
Pythagoras's theorem, $\mathrm{p}^{2}=2 \mathrm{q}^{2}$. Now it is trivially true that $\mathrm{p}^{2}$ contains an even number of prime factors, for it is clear that in pp every prime factor must occur twice or a multiple of two times. The same is true of $\mathrm{q}^{2}$, so that $2 \mathrm{q}^{2}$ necessarily has to contain an odd number of prime factors (an even number plus one-viz. the number 2). Hence the two can never be the same.

A slightly less direct approach we find in Euclid. If the two line segments would be commensurable, there has to be a greatest common measure $m$. Say the diagonal is $\mathrm{p} m$ and the side $\mathrm{q} m$. Then $\mathrm{p}$ and $\mathrm{q}$ cannot both be even, for in that case $2 m$ would be a greater common measure, contrary to our assumption. But $\mathrm{p}^{2}$ clearly is divisible by $2\left(\mathrm{p}^{2} / 2=\mathrm{q}^{2}\right)$, therefore $p$ itself has to be divisible by 2 , say $p=2 r$. But then $2 q^{2}=4 r^{2}$, hence $q^{2}=2 r^{2}$, and q necessarily has to be even, too.

Although I do not know which version is closest to the 'real' history, I prefer the first, because it agrees more with Aristotle's statement and is purely arithmetical, not appealing to anything geometrical. Euclid's version incorporates already the shift to geometrical language initiated by Eudoxus. But also didactically it seems to me important that whereas Euclid speaks of the oddness or evenness of $\mathrm{p}$ and $\mathrm{q}$ themselves, the first version speaks about the oddness or evenness of the numbers of prime factors in $\mathrm{p}$ and $\mathrm{q}$ - the problem thus is shifted to a higher level of abstraction, that of properties of properties of numbers.

However this may be, the 'relation' between diagonal and side was called-very appropriately-irrational (not a numerical ratio), and therefore 'inexpressible' (illogical), inexpressible, that is, in the arithmetical language of the Pythagoreans.

Thus we face the following problem situation: is it possible to define the notion of a ratio in such a way as to enable us to express relations between magnitudes in a perfectly exact fashion even if these magnitudes are incommensurable?

Eudoxus solved this problem in the following manner. First a general definition of a ratio is given as follows: 'we say that magnitudes are in a certain ratio when, multiplied, they can exceed one another'.

Next a relation of identity is developed in the following manner. The areas of rectangles $\mathrm{A}$ and $\mathrm{B}$ with equal height $\mathrm{h}$ are in the ratio $\mathrm{a}: \mathrm{b}$ of their bases, for $\mathrm{A}=\mathrm{ah}$ and $\mathrm{B}=\mathrm{bh}$. If $\mathrm{a}$ and $\mathrm{b}$ are commensurable, there are integers $m$ and $n$ such that $\mathrm{A}: \mathrm{B}=\mathrm{a}: \mathrm{b}=m: n$. But if a and $\mathrm{b}$ are incommensurable, there are no integers $m$ and $n$ that fulfil this requirement. Then neither a:b nor A:B are 'expressible', although it is clear that even in this case the proportionality $\mathrm{A}: \mathrm{B}=\mathrm{a}: \mathrm{b}$ must hold! Ultimately, therefore, it concerns a statement about the equality of 'things' that are themselves inexpressible. This statement runs as follows (definition 5 of Euclid's Elements Book V):

\footnotetext{
Magnitudes are said to be in the same ratio, the first to the second and the third to the fourth when, if any equimultiples whatever be taken of the first and third, and any equimultiples whatever of the second and fourth, the former equimultiples alike exceed, are alike equal to, or alike fall short of, the latter equimultiples respectively taken in corresponding order.
}

In modern notation:

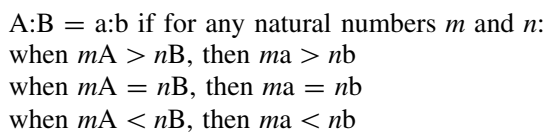

An order relation was introduced as follows:

$\mathrm{a}: \mathrm{b}>\mathrm{c}: \mathrm{d}$ when there are natural numbers $m$ and $n$ such that $m \mathrm{a}>n \mathrm{~b}$ and $m \mathrm{c}<n \mathrm{~d}$ 
Note that the notion of a ratio has been transformed from a numerical to a geometrical concept: the definition does not refer to numbers but to magnitudes with a dimension: lengths, areas or volumes. The definition says in effect that there can only be a ratio between magnitudes of the same dimension: a length, however multiplied, can never exceed an area, however small, etc.

Eudoxus's approach shows in brilliant clarity what the expansion of concepts implies. The notion of 'ratio' was stretched in order to overcome its earlier restrictions. What was unproblematic in the older theory was preserved as a special case (the commensurable case above). From the perspective of this new concept, incommensurability could now be regarded as a curiosity that could not hamper the further development of the theory. It implied, however, the complete downfall of the Pythagorean arithmetical cosmology. Henceforth the world was conceived as a geometric structure erected on a geometric basis. Non-numerical, dimensioned magnitudes (lengths, areas, volumes) were now considered the ultimate elements of visible reality. Euclid's 'Elements' fulfilled and consolidated this programme of the autonomous development of geometry, without the assumption of commensurability or rationality.

\section{Dedekind}

In the nineteenth century, the German mathematician Dedekind almost literally used Eudoxus's theory of proportionality as a basis for the construction of the irrational numbers out of the rational numbers. It was in teaching the calculus that he felt the need of a solid foundation especially of the notion of continuity, which he found in 1858 , under the inspiration of Eudoxus's theory. As he wrote: 'This same most ancient conviction has been the source of my theory' (Dedekind 1963 [1887], p. 40).

He remarked that a given point $\mathrm{P}$ divides the line in two pieces such that every point of the one piece lies to the left of every point of the other, and then continues: 'I now find the essence of continuity in the reversal, so in the following principle: if all points on a line are partitioned in two classes such that every point of the first class lies left of every point of the second class, then there exists one and only one point that generates the said partition.' This unique point he called a Schnitt (cut). Dedekind recognised that it was precisely Eudoxus's definition of proportionality that induced in the set of positive rational numbers a partition into two classes $\mathrm{A}_{1}$ and $\mathrm{A}_{2}$ such that for all $n, m$ in $\mathrm{A}_{1}$ we have $m \mathrm{~A} \geq n \mathrm{~B}$ and for all $n, m$ in $\mathrm{A}_{2}$ we have $m \mathrm{~A}<n \mathrm{~B}$. If we now interpret $\mathrm{A}: \mathrm{B}$ - entirely contrary to Greek ideas about number and proportion-as a fraction, then for $n, m$ in $\mathrm{A}_{1}$ we would have $n / m \leq \mathrm{A} / \mathrm{B}$, and for $n, m$ in $\mathrm{A}_{2}$ we would have $n / m>\mathrm{A} / \mathrm{B}$. If $\mathrm{A}_{1}$ contains a greatest (rational) element $r$, then the pair $\left(\mathrm{A}_{1}, \mathrm{~A}_{2}\right)$ corresponds with the rational number $r$. If $\mathrm{A}_{1}$ has no greatest element, then we can make the pair $\left(\mathrm{A}_{1}, \mathrm{~A}_{2}\right)$ correspond to 'something new' - an irrational number. In this way Dedekind arrived at the idea of introducing abstractly a partition of the field of rationals in two classes $\mathrm{A}_{1}$ and $\mathrm{A}_{2}$, which partition by definition possesses the characteristic properties ensuing from Eudoxus's theory of proportionality, viz. identity and order relations for the field of real (rational and irrational) numbers.

This necessarily somewhat oversimplified account must suffice here to make the point that even after more than two millennia the objective content of Eudoxus's theory was invoked as a way of 'inventing' a new kind of numbers, quite independently from whatever Eudoxus or any other Greek might have had in mind, 'really'. This is the important difference with the so-called 'real history'. It was from the objective content only that Dedekind 'learned' his deep insight into the continuity of the real line. 


\section{Concluding Remarks}

Questions pertaining to the body of mathematical knowledge must be distinguished from issues regarding social and cultural concerns or interests. Indeed, it is only from the consideration of mathematics as a body of objective knowledge that its psychological, social and cultural aspects can be placed in their proper perspective. Therefore the analysis of objective problem situations has priority over the psychology of mathematicians or the sociology of mathematical communities. Although it is obvious that the development of mathematics depends on the participation of human mathematicians and their communities, nevertheless mathematical concepts bear relationships to each other that are independent of whether individual mathematicians are aware of them, or whether the mathematical community as an institution recognizes them.

Objective questions about the contents of mathematical theories must be distinguished from questions about mental attitudes, psychological responses, and the like, of the historical figures who held these theories. Mathematicians deal all the time with objective (world-3) contents, which are in many respects autonomous and can exert important feed-back effects upon the world- 2 of mental states and processes and even the physical world-1. Of course, both sets of questions - about knowledge in the objective and in the subjective sense-can be entirely legitimate; what is not legitimate, however, is to confound the objective dimension with the subjective, and to imagine that exclusive concern with the latter will suffice to all intents and purposes. For the purpose of history-based learning about mathematical in-issues, concern with the objective, rationally reconstructible dimension is indispensable.

Open Access This article is distributed under the terms of the Creative Commons Attribution Noncommercial License which permits any noncommercial use, distribution, and reproduction in any medium, provided the original author(s) and source are credited.

\section{References}

Charalambous, Y., Panaoura, A., \& Philippou, G. (2009). Using the history of mathematics to induce changes in preservice teachers' beliefs and attitudes: Insights from evaluating a teacher education program. Educational Studies in Mathematics, 71, 161-180.

Dedekind, R. (1963) [1887]. Essays on the theory of numbers. New York: Dover.

Fauvel, J., \& Van Maanen, J. (Eds.). (2000). History in mathematics education: The ICMI study. Dordrecht: Kluwer.

Fried, M. N. (2001). Can mathematics education and history of mathematics coexist? Science \& Education, $10,391-408$.

Gulikers, I., \& Blom, K. A. (2001). A historical angle, a survey of recent literature on the use and value of history in geometrical education. Educational Studies in Mathematics, 47, 223-258.

Jankvist, U. T. (2009). A categorization of the "whys" and "hows" of using history in mathematics education. Educational Studies in Mathematics, 71, 235-261.

Jankvist, U. T. (2010). An empirical study of using history as a goal. Educational Studies in Mathematics, 74, 53-74.

Popper, K. R. (1972). Objective knowledge: An evolutionary approach. Oxford: Oxford University Press. 